\title{
Novel Nonsense Mutation in SLC39A13 Initially Presenting as Myopathy: Case Report and Review of the Literature
}

\author{
Maja Dusanic a,c, e Gabriele Dekomien ${ }^{a, c}$ Thomas Lücke ${ }^{\text {b, c }}$ \\ Matthias Vorgerd ${ }^{c, d}$ Joachim Weis ${ }^{f}$ Joerg T. Epplen ${ }^{a, c, e}$ Cornelia Köhler $^{b, c}$ \\ Sabine Hoffjan ${ }^{a, c}$ \\ a Department of Human Genetics, Ruhr-University Bochum, 'b Department of Neuropediatrics, University Children's \\ Hospital, Ruhr-University Bochum, ' Center for Rare Diseases Ruhr (CeSER), and d Department of Neurology,

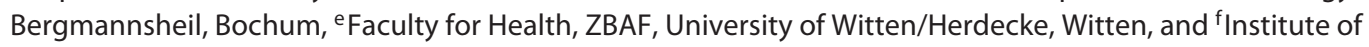 \\ Neuropathology, RWTH Aachen Medical School, Aachen, Germany
}

\section{Keywords}

Exome sequencing $\cdot$ Next-generation sequencing ·

SCD-EDS · SLC39A13 - Spondylocheirodysplasia EDS-like syndrome $\cdot$ ZIP13

\begin{abstract}
Myopathies comprise a heterogeneous group of disorders characterized by variable phenotypes. The increasing use of next-generation sequencing allows identification of the causative genes in a much higher percentage of patients with hereditary muscle disorders and also illustrates a considerable degree of overlap with other clinical entities, including connective tissue disorders. Here, we present a 14-year-old German patient who was initially suspected to suffer from myopathy based on his clinical, radiological, and muscle biopsy findings. Exome sequencing revealed a novel homozygous nonsense mutation in the SLC39A13 gene, causative for spondylocheiro dysplastic Ehlers Danlos syndrome (SCD-EDS), suggesting a connective tissue disorder. Including our patient, only 9 affected individuals from 4 fam-
\end{abstract}

\section{KARGER}

(C) 2018 S. Karger AG, Basel

E-Mail karger@karger.com

www.karger.com/msy ilies have been described for SCD-EDS so far. The previously reported patients did not show obvious evidence of myopathy, suggesting a broader clinical presentation than originally suspected. We summarize herein the current knowledge on clinical features as well as pathophysiological pathways for this rare connective tissue disease and discuss the high degree of clinical overlap between myopathic and connective tissue disorders.

(c) 2018 S. Karger AG, Basel

The term myopathy comprises a wide spectrum of muscular diseases with variable phenotypic presentation, characterized by muscle weakness, atrophies and/or myalgias, muscle stiffness, cramps, and an onset ranging between birth and adulthood [Mattle and Mummenthaler, 2013]. Hereditary myopathies have proven to be highly heterogeneous, which made the establishment of the cor-

\section{C.K. and S.H. contributed equally to this work.}

Sabine Hoffjan, MD

Department of Human Genetics, Ruhr-University Bochum

Universitätsstr. 150

DE-44801 Bochum (Germany)

E-Mail sabine.hoffjan@ruhr-uni-bochum.de 
rect diagnosis rather difficult before the era of next-generation sequencing (NGS) [Ravenscroft et al., 2017]. With the increasing use of NGS technologies, it became obvious that a substantial part of patients carry mutations in genes associated with a myopathy subtype different from what has been clinically suspected [Nigro and Savarese, 2016; Savarese et al., 2016]. Additionally, a high amount of clinical overlap with other disease entities, for example connective tissue disorders, was evident, which had already been described before NGS methods were well-established in the diagnostic approach [Voermans et al., 2008].

We present a 14-year-old German boy of healthy consanguineous parents who was initially diagnosed with myopathy. Exome sequencing revealed a novel homozygous nonsense mutation in the SLC39A13 gene associated with the spondylocheiro dysplastic form of Ehlers Danlos syndrome (SCD-EDS) [Guinta et al., 2008]. So far, only 8 other patients from 3 families have been described for SCD-EDS. Starting from our case presentation, we summarize the current knowledge on clinical features as well as the genetic background and pathophysiological pathways for this rare connective tissue disease and discuss the high degree of clinical overlap between myopathic and connective tissue disorders.

\section{Case Report}

After uncomplicated pregnancy, our male index patient was born spontaneously at full term to healthy consanguineous German parents. He showed sensitive skin and was diagnosed with atopic dermatitis. The patient displayed a normal psychomotor development until the age of 1 year, when a delay in motor milestones became apparent: he did not crawl and started to walk independently at 18 months with a precarious gait. At the age of 4 years, he underwent his first thorough clinical evaluation showing walking difficulties, reduced endurance, enlarged calves, externally rotated flat feet, and ankle pain. At that point, mild dysmorphic facial features were visible. However, extensive neuropediatric evaluation did not reveal the etiology of the symptoms.

The patient was referred to the Neuromuscular Clinic of our Children's Hospital at the age of 11 years, still complaining about limited physical function and exercise-induced myalgias. His basic activities of daily life were not affected, but compared to his peers, moderate and prolonged physical activity led to fatigue and weakness suggesting a (neuro-) muscular disorder. MRI scans were suggestive for a myopathy, and a subsequent muscle biopsy showed unspecific myopathic signs supporting this diagnosis (see below). His musculoskeletal complaints appeared static over the years. Neuropediatric examination revealed mild, predominantly distal muscular weakness (MRC 4/5), manifesting with moderate limitations in the extensor muscles in his upper extremities and the flexor muscles in the lower extremities.
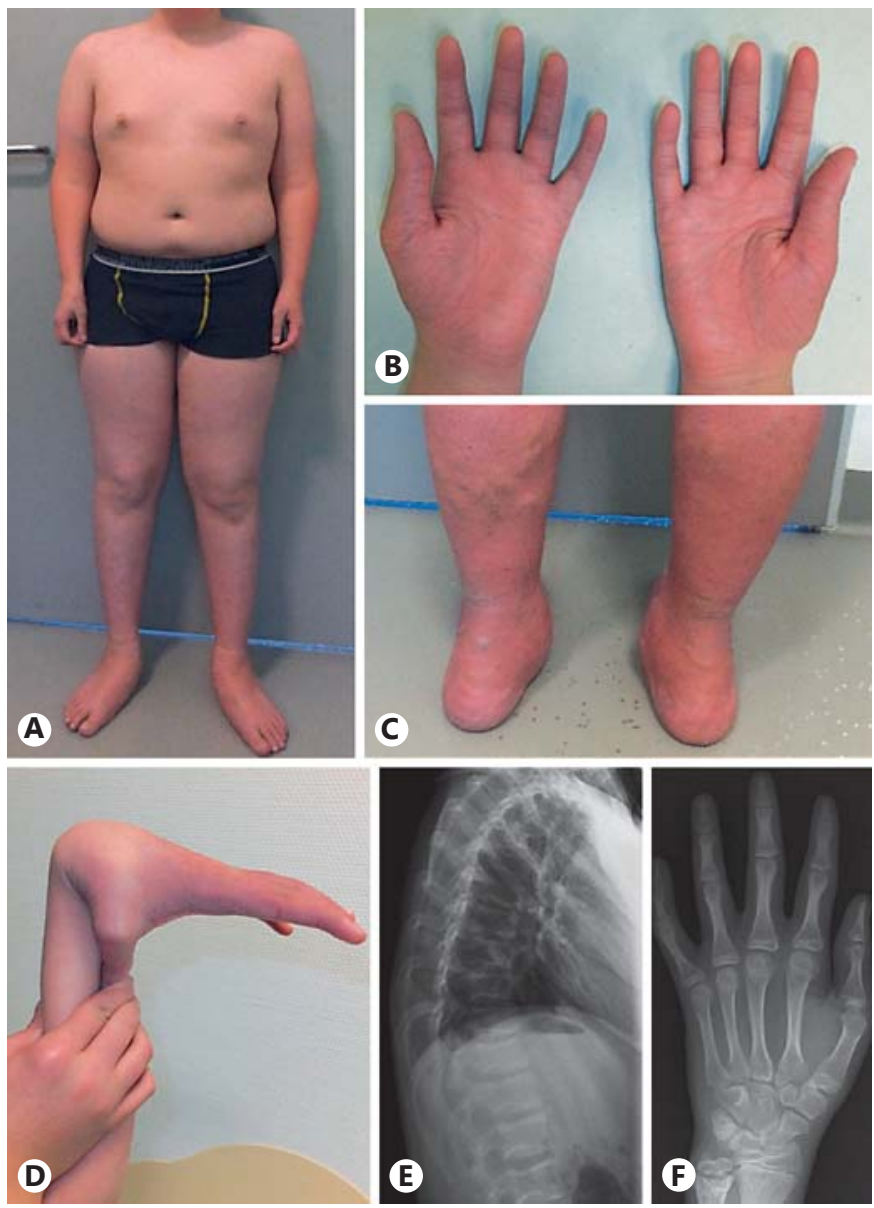

Fig. 1. Phenotypic and radiological features of the affected individual. A Disproportional body measurements with small hands, short arms and trunk, long legs, forearm and calves' atrophy. B Tapering fingers, wrinkled palms, hypothenar and thenar atrophy. C Externally rotated flat feet and varicosis of the lower extremity. D Hypermobility of joints. E Mild flattening of the vertebral bodies. F Slight shape variations, irregular epiphyses of the proximal and distal phalanges, broadened ulnar epiphysis.

At that time, a waddling gait and a positive Gowers sign were obvious, running was barely possible, walking on tiptoes was impossible. After frequent participation in weight training, a follow-up examination 3 years later revealed some improvements of his myopathic complaints. His heel and toe walking improved, and he was able to run. However, atrophy of the calves and forearms was obvious as well as an ungainly monopedal jumping underlining the myopathic component of his symptom complex. Tendon reflexes were retained, muscle tone was normal, and no sensory deficit was noted. Throughout the years, obesity with a BMI of 25.6 as well as a short and unproportional stature (below the 3 rd percentile for his age with short arms, trunk height: 155.2 $\mathrm{cm}$, sitting height: $75 \mathrm{~cm}$, and arm span: $155 \mathrm{~cm}$ ) became apparent (Fig. 1A). Dysmorphic facial features including a round face with a flat facial profile, hypertelorism, grayish sclerae, protuber-
Nonsense Mutation in SLC39A13

Presenting as Myopathy
Mol Syndromol 2018;9:100-109

DOI: $10.1159 / 000485881$
101 
Fig. 2. MRI and muscle biopsy findings. A, B MRI of the lower extremity at the age of 14 years displays bilateral, symmetrical lipomatous atrophy of the vastus mediales, rectus femoris muscles $(\mathbf{A})$ and the soleus, peroneus as well as feet extensor muscles (B). C H\&E staining of the muscle biopsy shows hypotrophic muscle fibers. Scale bar, $40 \mu \mathrm{m}$. D Electron microscopy reveals low density of endomysial collagen (black arrows) and a ring fiber with circular myofibrils (white arrows). Scale bar, $1 \mu \mathrm{m}$. E Membrane-bound accumulations of glycogen granules and autophagic membranous material (black arrow) as well as focal widening of the sarcoplasmatic reticulum (white arrows) are shown. Scale bar, 0.5 $\mu \mathrm{m}$. F Normal muscle fibers and endomysial extracellular matrix including bundles of collagen fibers (arrows) in an age-matched control muscle. Scale bar, $0.5 \mu \mathrm{m}$.
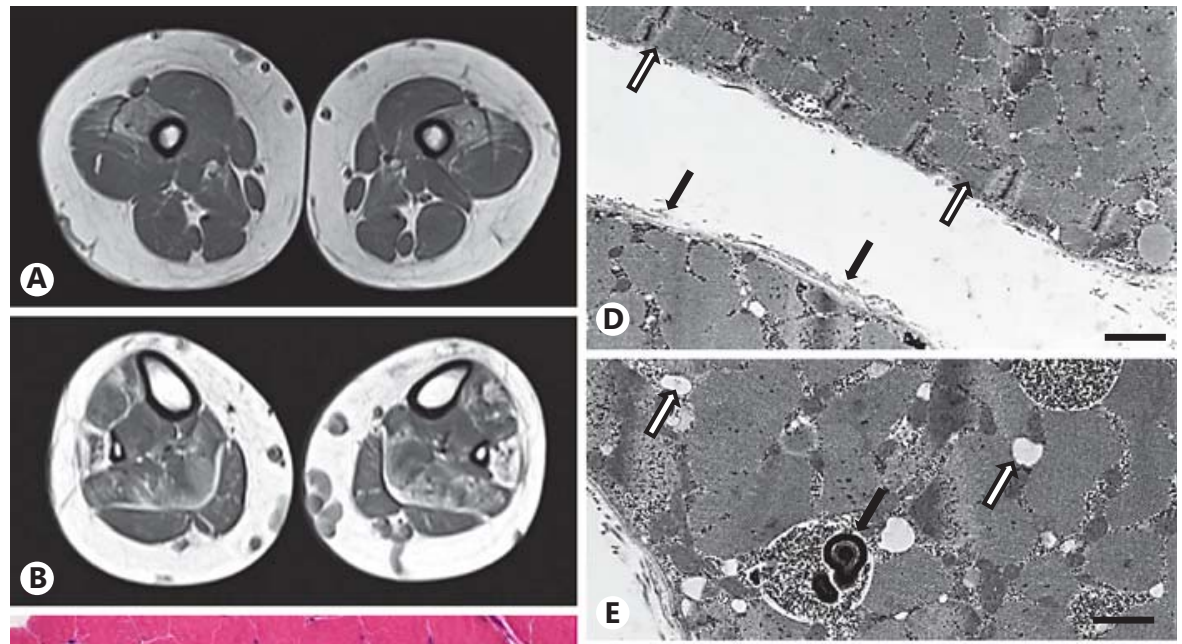

B
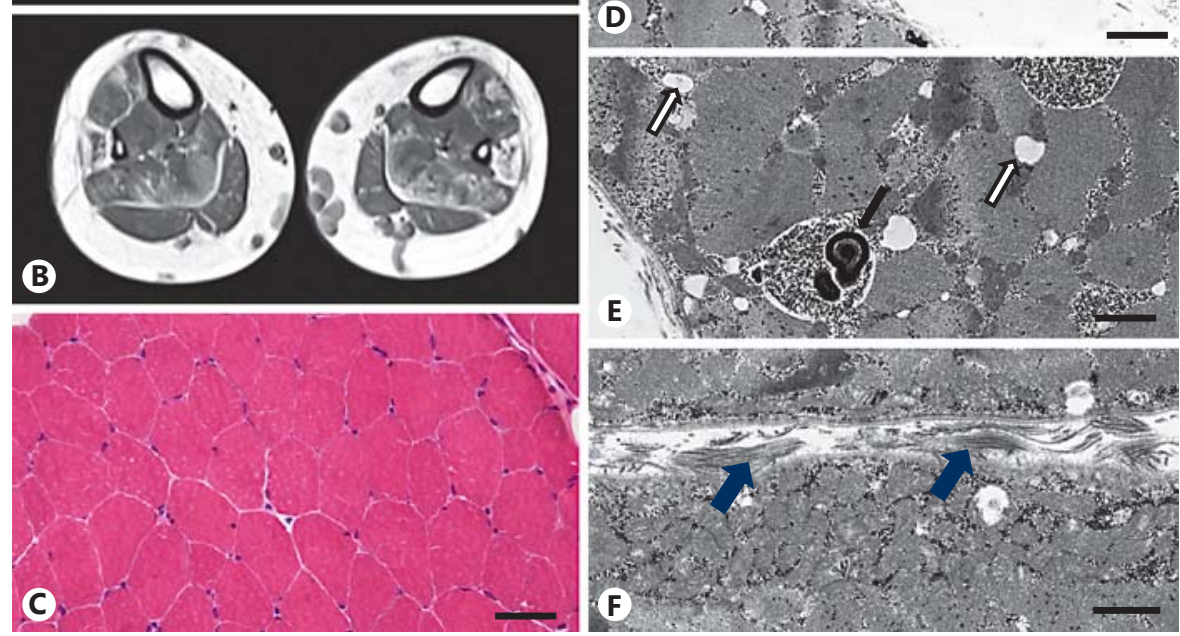

ant eyes, wide nasal base with a low nasal bridge and high-arched palate were present. A diagnosis of dysgnathia required orthodontic treatment for several years. Furthermore, the patient showed sensitive and scaling skin on his distal extremities with a cigarette paper-like appearance requiring frequent skin treatment. During physical examination the blood pressure cuff caused an upper arm hematoma, and easy bruising was confirmed by the patient. His fingers, hands, and feet were tapering with wrinkled palms (Fig. 1B); his thumbs were placed proximally coupled with thenar and hypothenar muscular atrophy and considerable small joint hypermobility (Fig. 1D). On the lower extremities as well as on the abdominal skin, varicose veins were visible (Fig. 1C). Joint contractures or cutis laxa were not obvious, but conservatively treated bilateral pedes plani (Fig. 1A, C), hyperlordosis, and scoliosis were noted. The patient exhibits normal intellectual development; his 12-year-old sister is healthy, and no family history of inherited or acquired neuromuscular disorders was reported.

\section{Methods and Results}

\section{Clinical Findings}

Blood and urine tests in our patient revealed normal results for creatine kinase, lactate, creatinine, carnitine, acylcarnitine, amino acids in plasma and urine, organic acids in urine as well as biotinidase activity. Echocardiography, electrocardiography, and pulmonary function testing gave regular results. Nerve conduction velocities were within the normal range. From a dermatological consultation, a mild form of ichthyosis vulgaris was suspected accompanied by aseptic eczema.
Since an elevated urinary LP/HP (lysyl pyridinoline/hydroxylysyl pyridinoline) ratio had been described for SCD-EDS in the literature [Fukada et al., 2008; Guinta et al., 2008], we measured this ratio in our patient by performing acid hydrolysis and reverse phase high performance liquid chromatography with fluorometric detection. The LP/HP ratio was found to be 0.27 , which is only slightly elevated.

\section{Radiological Findings}

Musculoskeletal ultrasound of thigh and calf muscles at 4 years of age showed regular echo patterns. Radiological evaluations of the thoracic and lumbal vertebrae at the age of 4 years were recorded as normal. However, at the age of 14 years, lumbal lordosis was noted accompanied by a mild flattening of thoracic vertebrae (Fig. 1E). Further, mild alterations of shape and flat epiphyses of the phalanges as well as an enlarged epiphyseal plate of the distal ulna were noted (Fig. 1F).

MRI scans of the lower extremities, performed at 9 and 14 years of age, revealed progressive bilateral and symmetrical lipomatous muscle alterations and a slight muscle edema in distal, predominantly anterior thigh compartments (Fig. 2A). Lipomatous atrophy was also present in the soleus, peroneus, and feet extensor muscles (Fig. 2B). The radiological pattern was interpreted as typical for either metabolic or classical structural myopathy. In addition, prominent dilatation of superficial varicose veins was detected. Cranial MRI scans were according to age.

\section{Muscle Biopsy Findings}

Muscle biopsy from the vastus lateralis muscle was performed at the age of 11 years. A nonspecific myopathy pattern with some disseminated hypotrophic muscle fibers (type 1: $12-50 \mu \mathrm{m}$; type 2: $18-49 \mu \mathrm{m}$; normal: $40-80 \mu \mathrm{m}$ ) was observed (Fig. $2 \mathrm{C}$ ). There 
Fig. 3. Identification of the c. $830 \mathrm{G}>\mathrm{A}$ mutation in the SLC39A13 gene through exome sequencing and confirmation with Sanger sequencing. The index patient is homozygous for the nonsense mutation, while the unaffected parents are heterozygous carriers.

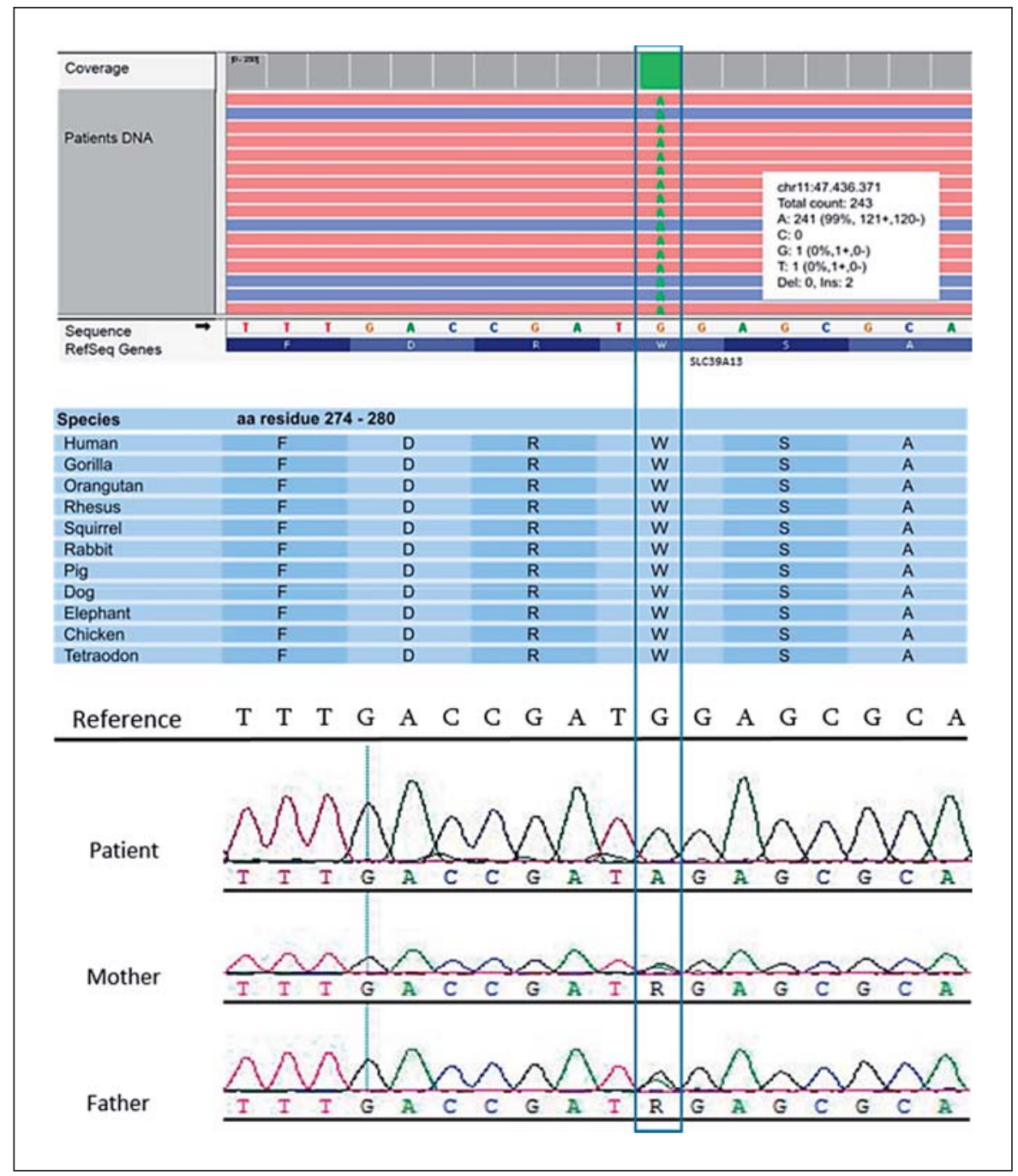

was no evidence of inflammatory, dystrophic, or structural changes in the myofibrillar architecture. ATPase staining showed a slight predominance of type 2 fibers, only one small group of type 1 fibers, and no oxidative enzyme reaction abnormalities (not shown).

Electron microscopy revealed markedly reduced endomysial collagen fibrils and several ring fibers (Fig. 2D), widening of sarcoplasmic reticulum cisterns and considerable focal intermyofibrillar accumulations of glycogen granules in membrane-bound vacuoles. In few of these vacuoles, myelin-like membranous osmiophilic material indicative of impaired autophagy was found (Fig. 2E) in comparison to normal tissue (Fig. 2F).

\section{Genetic Analyses}

Chromosomal analysis and molecular karyotyping gave normal results. As Ullrich congenital muscular dystrophy (UCMD) had originally been suspected, sequencing of the collagen VI genes was conducted, revealing a paternally inherited heterozygous missense mutation in exon 12 of the COL6A2 gene which likely represents a rare variant rather than a pathogenic mutation. Further- more, based on electron microscopy findings, the ABHD5 and PNPLA2 genes (causative for Charnarin-Dorfman syndrome) were analyzed, but pathogenic mutations were not identified. Therefore, a diagnostic gene panel for structural myopathies including 9 genes (RYR1, ACTA1, CFL2, TPM3, TPM2, TNNT1, $K B T B D 13, B I N 1$, and MTM1) was performed using the Ion Torrent $^{\mathrm{TM}}$ technology (Life Technologies, Carlsbad, CA, USA). Since no pathogenic mutation was discovered, we expanded genetic testing by applying exome sequencing based on the abovementioned technology. For enrichment of the library, we used the Ion OneTouch ES instrument (Life Technologies). The sequencing displayed a mean coverage of 121 -fold for all exons, while $89 \%$ of them were covered at $>20 \times$. Conversion into BAM files and sequence alignment against the human reference (GRCh37/hg19) was performed with the Ion Torrent Suite Software V5.0.5. Subsequent analysis with the NextGENe V2.4.2 software (SoftGenetics, State College, PA, USA) revealed a homozygous nonsense mutation in exon 8 of the SLC39A13 gene (c.830G>A, p.W277*) (Fig. 3). This mutation is located in a region that is highly conserved among vertebrates (Fig. 3) and was not found in databases such as dbSNP 


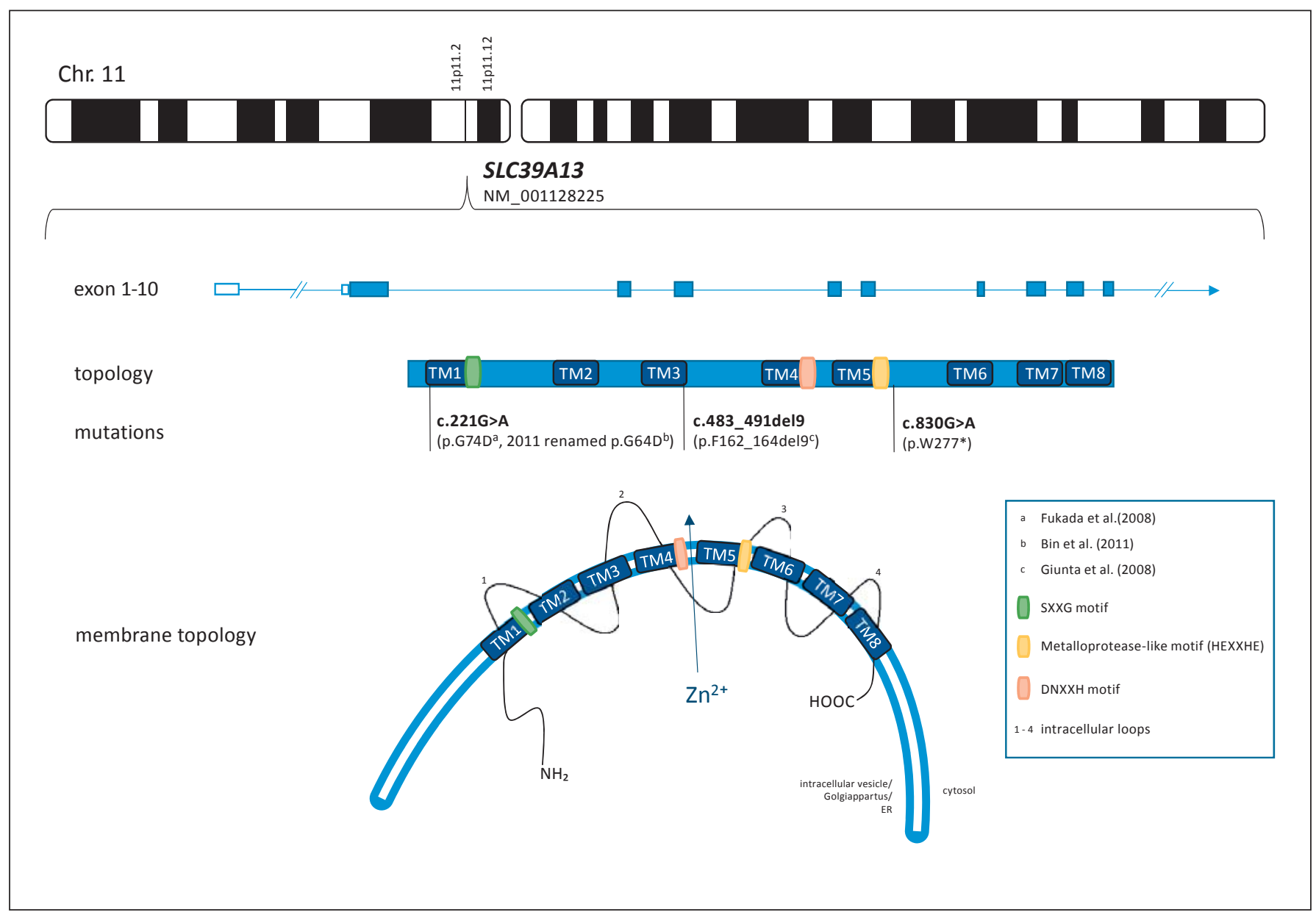

Fig. 4. Localization, genomic structure and schematic (membrane) topology of the SLC39A13 gene/ZIP13 including the mutations described in the literature so far. The c. $221 \mathrm{G}>\mathrm{A}$ mutation was renamed by Bin et al. [2008] and relocated into the first transmembrane domain. SLC39A13 is predicted to be composed of 10 exons

(Single Nucleotide Polymorphism Database), ExAc (Exome Aggregation Consortium Browser), or the 1000 genomes project. In silico prediction programs such as the likelihood ratio test [Chun and Fay, 2009] and MutationTaster [Schwarz et al., 2014] suggested a disease-causing effect of the mutation with a high probability, predicting it to result in the loss of several topographical regions (see Fig. 4). Sanger sequencing confirmed both parents to carry the mutation in heterozygous state (Fig. 3).

\section{Discussion}

\section{SCD-EDS, New Classifications, and Overlap}

Syndromes

Together with our patient, 9 individuals from 4 families have been reported in the literature so far that carry coding for 8 transmembrane domains and 4 intracellular loops. Functionally important motifs include HEXXHE and DNXXH as possible zinc-binding sites and SXXG as an important component in protein stability [Taylor and Nicholson, 2003; Bin et al., 2014; Kambe et al., 2015b]. TM, transmembrane domain.

disease-causing mutations in the SLC39A 13 gene. Guinta et al. [2008] first identified an identical homozygous 9-bp deletion (c.483_491del9, p.F162_164del) in 6 patients of 2 different consanguineous families. The combination of skeletal (spondylo), hand (cheiro), and skin abnormalities in these patients led the authors to propose a new and rarely occurring subtype of EDS with an autosomal recessive mode of inheritance, the spondylocheiro dysplastic Ehlers-Danlos- (like) syndrome (OMIM 612350). In comparison to the closely related EDS type VI (OMIM 225400), they pointed out 8 characteristic features for SCD-EDS; these include growth retardation, sensitive and transparent skin, hand and joint abnormalities, bone deformities with radiological pathologies, protuberant gray-bluish sclerae, and abnormal pyridinolines (urinary 
Table 1. Current classifications for SCD-EDS and clinical findings in the affected individuals reported in the literature including the current case report

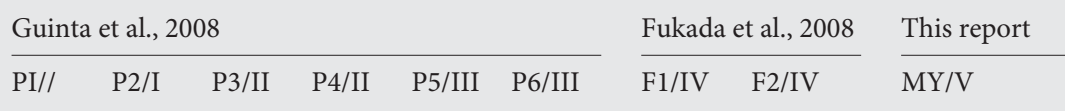

EDS classification 2017

spEDS criteria

Major criteria

Short stature

Muscle hypotonia

Bowing of limbs

Minor criteria

Skin abnormalities

Pes planus

Delayed motor development

Osteopenia

Delayed cognitive development

$\begin{array}{lllllllll}+ & + & + & + & + & + & + & + & + \\ ? & ? & ? & ? & ? & ? & + & + & + \\ +\mathrm{m} & \text { n.r. } & + & + & + & + & + & + & + \\ & & & & & & & & + \\ + & + & + & + & + & + & + & + & + \\ + & + & + & + & + & + & ? /+ & ? & + \\ ? & - \text { fy } & + & - & + & - & ? & ? & - \\ + & + & + & + & - & + & + & + & -\end{array}$

SCD-EDS specific

Protuberant eyes with bluish sclerae

Hands with finely wrinkled palms

Atrophy of the thenar muscles and tapering fingers

Hypermobility of distal joints

Characteristic radiologic findings

$\begin{array}{lllllllll}+ & + & + & + & +\mathrm{fy} & + & + & + & + \\ + & + & + & + & + & + & + & ? /+ & + \\ + & + & + & + & + & + & ? /+ & ? & + \\ +\mathrm{m} & +\mathrm{m} & +\mathrm{m} & +\mathrm{m} & + & + & + & + & + \\ + & + & + & + & + & + & + & + & +/-\end{array}$

Guinta et al., 2008 criteria

\begin{tabular}{|c|c|c|c|c|c|c|c|c|c|c|}
\hline 1 & Short stature & + & + & + & + & + & + & + & + & + \\
\hline \multirow[t]{6}{*}{2} & Skin abnormalities & & & & & & & & & \\
\hline & Hyperelastic & $+\mathrm{m}$ & $+\mathrm{m}$ & $+\mathrm{m}$ & $+\mathrm{m}$ & + & + & - & - & - \\
\hline & Thin, cigarette paper like & + & + & + & + & + & + & + & + & + \\
\hline & Easily bruisable & + & - & + & + & $+/ \#$ & + & + & + & + \\
\hline & Atrophic scars & + & + & + & + & -hy & + & $?$ & $?$ & - \\
\hline & Varicosis & + & - & $?$ & + & + & + & + & + & + \\
\hline
\end{tabular}

3-5 Finger, hand, and joint abnormalities

Hypermobility

Contractures

Tapering fingers

Wrinkled palms

Thenar/hypothenar atrophy

$>$ metaphyseal regions

$\begin{array}{lllllllll}+\mathrm{m} & \mathrm{n} . \mathrm{r} . & + & + & + & + & + & + & + \\ +\mathrm{m} & \mathrm{n} . \mathrm{r} . & + & - & + & + & ? & ? & - \\ + & + & + & +\mathrm{m} & + & + & ? & ? & + \\ + & + & + & + & +\mathrm{m} & + & + & ? /+ & + \\ + & + & + & + & + & + & ? /+ & ? & + \\ - & - & + & + & + & + & ? & ? & ?\end{array}$

\begin{tabular}{|c|c|c|c|c|c|c|c|c|c|c|}
\hline 6 & Protuberant/gray-bluish eyes & + & + & + & + & $+\#$ & + & + & + & + \\
\hline \multirow[t]{4}{*}{7} & Radiological findings & & & & & & & & & \\
\hline & Platyspondyly & + & + & + & + & + & + & + & + & $+/-$ \\
\hline & Osteopenia & + & + & + & + & - & + & + & + & - \\
\hline & >ends of tubular hand bones & + & + & + & + & + & + & $?$ & $?$ & $+/-$ \\
\hline
\end{tabular}

* Presented as HP/LP ratio in this report. fy, first year; m, mild; hy, hypertrophy; n.r., not reported; SCD-EDS, spondylocheiro dysplastic Ehlers Danlos syndrome; \#, improved; +, present; -, absent; ?, unknown. ?/+, not clearly stated in the text, but may be deducted from the figures in the respective report; $+/-$, slightly abnormal findings.

LP/HP ratio of approximately 1.0) [Guinta et al., 2008] (Table 1). Fukada et al. [2008] identified 2 additional patients of possibly related Portuguese parents with a homozygous missense mutation in the SLC39A13 gene
(c.221G>A, p.G74D) presenting 6 of the abovementioned 8 clinical features. None of these patients displayed a BMI $>25$ or obvious features of a myopathy as seen in our patient. However, since both parents of our patient have 
increased BMI values (father 31.4; mother 29.7), the high BMI could also be unrelated to SCD-EDS.

EDS in general comprises a highly heterogeneous group of heritable connective tissue disorders. Over the past few years, several new pathogenic variants and/or novel disease genes have been detected mainly through NGS, and a new international classification of EDS was published in 2017 [Malfait et al., 2017]. Within this classification, SCD-EDS together with 2 related clinical entities, $\beta 3$ GalT6 and $\beta 4$ GalT7 deficiency, form the EDS subtype "spondylodysplastic form of EDS (spEDS)" [Brady et al., 2017]. Short statue, muscle hypotonia, and bowing of limbs are considered major criteria in spEDS, while skin hyperextensibility, pes planus, osteopenia, and delayed motor and cognitive development rate as minor criteria [Malfait et al., 2017]. Our patient fulfills at least 6 of 8 of the SCD-EDS features initially defined by Guinta et al. [2008] (Table 1). The radiological abnormalities in the spine and the hands appear to be rather mild but still overall consistent with the features presented in the literature. However, our patient does not have a strongly elevated LP/HP ratio, suggesting that this measurement may be less common in patients with SCD-EDS. Interestingly, this criterion is not part of the recently published EDS classification for SCD-EDS, of which our patient fulfills 3 major and 7 of 10 minor criteria (Table 1). The only mildly reduced ratio could reflect less pronounced bone degradation in our patient, in accordance with only mild radiological findings. While revising this article, however, our patient was diagnosed with a Weber $\mathrm{C}$ ankle fracture after minor trauma, which may support a form of osteopenia.

Muscular hypotonia and/or delayed motor development have been described as common neuromuscular features in spEDS [Malfait et al., 2017]. However, although all of the described patients presented with neuromuscular symptoms such as thenar/hypothenar atrophy, weakness of hands, pathological gait [Guinta et al., 2008], or muscular hypotonia after birth [Fukada et al., 2008], none of them had further histological or electrophysiological evaluation regarding a myopathy. Our case report, therefore, underlines the presence of myopathic changes in SCD-EDS, proposing a wider clinical presentation then assumed until now.

A clinical overlap between EDS and myopathy is wellknown as all EDS classifications contain neuromuscular symptoms [Beighton et al., 1998; Brady et al., 2017; Malfait et al., 2017] which are mostly interpreted as secondary manifestations of connective tissue disorders [Kirk et al., 1967; Royce and Steinmann, 2002; Castori and Voer- mans, 2014]. Furthermore, defective extracellular matrix (ECM) molecules are believed to disintegrate the muscle cells' structural stability [Voermans et al., 2008, 2009; Savasta et al., 2011; De Paepe and Malfait, 2012]. Based on our findings, SCD-EDS not only shows overlapping symptoms with the $\beta 3 \mathrm{GalT} 6$ and $\beta 4 \mathrm{GalT} 7$ deficiencies resulting in defective glycosaminoglycan regions [Okajima et al., 1999; Nakajima et al., 2013; Malfait et al., 2017], but also with "myopathic forms of EDS" based on COL12A1 mutations or muscular dystrophies such as Bethlem myopathy or UCMD caused by defective collagen VI [Voermans et al., 2008; Brady et al., 2017; Malfait et al., 2017]. Glycosaminoglycan and collagen VI both function as connective tissue elements, specifically as ECM components. Bethlem myopathy may present with skin features similar to the ones described in this case report, including cigarette paper-like appearance or scaling skin [Camacho et al., 2001; Kirschner et al., 2005; Yonekawa and Nishino, 2015], and UCMD patients often present with considerable small joint hypermobility within their myopathy disorder [Yonekawa and Nishino, 2015]. UCDM was even considered as a potential diagnosis for our patient, but not confirmed through genetic testing. On the other hand, small joint hypermobility, fragile translucent skin, and easy bruising should prompt further evaluation with regard to connective tissue disorders in patients with suspected myopathy. Nowadays, however, exome sequencing would probably have been performed earlier in the disease course, thus establishing a diagnosis without the sequencing of several single genes in advance.

In conclusion, the observed myopathic changes in skeletal muscle in a patient with SCD-EDS suggest that this rare EDS subtype is based on defective or dysregulated ECM components not only in connective [Hirose et al., 2015], but also in muscular tissue cells. The increasing use of NGS technologies will certainly reveal further cases in which a clear clinical distinction between primary myopathic and connective tissue disorders is challenging and may only be resolved by genetic evaluation. Of course, we cannot exclude that our patient may carry additional, e.g., modifying, variation in genes involved in muscular pathophysiology, such as glycogen storage or autophagy, but at least from exome sequencing such variation has not been obvious.

\section{SLC39A13}

The gene mutated in SCD-EDS, SLC39A13, is $1.1 \mathrm{~kb}$ in size, located in chromosome 11 and comprises 10 exons. It encodes the zinc transporter protein Zrt- and Irt-like protein 13 (ZIP13) which forms a homo-dimer consisting
106

Mol Syndromol 2018;9:100-109 DOI: $10.1159 / 000485881$
Dusanic/Dekomien/Lücke/Vorgerd/Weis/ Epplen/Köhler/Hoffjan 
of 8 transmembrane domains and 4 intracellular loops (Fig. 4) [Bin et al., 2011]. ZIP13 belongs to one of 2 main groups of zinc transporters (ZnT/SLC30A and ZIP/ SLC39A) confirmed to participate in maintaining cellular zinc homoeostasis [Jeong and Eide, 2013; Kambe et al., 2015a; Kimura and Kambe, 2016; Hara et al., 2017]. In contrast to Zn transporters, ZIP family members operate as influx transporters moving zinc from extracellular fluids or intracellular organelles into the cytosol. Zn transporters have also been proven to participate in intracellular zinc-related signaling [Hirano et al., 2008; Fukada et al., 2013; Hara et al., 2017]. Imbalances in cellular zinc distribution lead to a diversity of symptoms (e.g., toxicity, copper or zinc deficiency syndromes) presenting with mild to severe or even life-threatening forms [Bin et al., 2011; Hara et al., 2017].

While the 2 previously known mutations localize in one of the 8 highly hydrophobic transmembrane domains, our patient's homozygous nonsense mutation (c.830G $>$ A, p.W277*) results in premature termination of translation and presumably loss of transmembrane domains 6-8, including the third and fourth intracellular loop as well as the C-terminus (see Fig. 4). Not much is known about these 2 loops; however, the second intracellular loop has been suggested to function as a zinc-binding site [Bin et al., 2011]. Supplementary studies are warranted to further evaluate the role of these regions for physiological functioning of the ZIP13 protein as well as their involvement in SCD-EDS pathogenesis.

\section{Current Knowledge of SCD-EDS Pathogenesis}

Giunta et al. [2008] suspected the c.483_491del9 mutation to cause abnormal protein folding, resulting in "zinc trapping" in intracellular organelles which may lead to an inhibition of iron-dependent collagen hydroxylases. Since their analysis predicted the ZIP13 protein to be localized most likely in the endoplasmic reticulum (ER), they expected high zinc concentrations therein to cause an alteration in collagen hydroxylation. However, hydroxylase activities extracted from patients' fibroblasts revealed normal enzyme function. Fukada et al. [2008] created a SLC39A13 knockout mouse model which demonstrated impairments of intracellular zinc concentration in connective tissue cells coupled with several abnormalities in bone, cartilage, teeth, and skin. The knockout mice closely resembled SCD-EDS, showing growth retardation, multiple bone deformities (including shortened long bones, progressive kyphosis as well as increased radiolucency and bone metabolism coupled with a reduced bone volume and density), craniofacial deformations

Nonsense Mutation in SLC39A13

Presenting as Myopathy with teeth abnormalities, and increased skin vulnerability. Unfortunately, skeletal muscle tissue was not reported to have been examined in the mouse model. Since high expression of the ZIP13 protein was observed in human cardiomyocytes, placental and skeletal muscle tissue [Jeong et al., 2012], and 60\% of zinc storage distribution occurs in skeletal muscle tissue [Jackson, 1989; Kambe et al., 2015b], additional investigations are warranted to clarify in which way mutations in the SLC39A13 gene affect skeletal muscle cells.

Fukada et al. [2008] localized the ZIP13 protein in the Golgi apparatus rather than in the ER and identified altered intracellular signaling pathways induced by transforming growth factor beta (TGF $\beta$ ) and bone morphogenetic protein 4 in the mouse model. Jeong et al. [2012], on the other hand, localized the ZIP13 protein predominantly in the lumen of intracellular vesicles, suggesting them to function as labile zinc-storing organelles. The latter authors hypothesized that the physiological function of ZIP13 consists in restoring low cytosolic zinc concentrations and that mutated ZIP13 proteins lead to zinc trapping inside the vesicles. Interestingly, our patient's muscle biopsy revealed an increased amount of accumulated glycogen granules, some of which were found in membrane-bound vacuoles, and changes of the sarcoplasmic reticulum, which may be linked to these pathomechanisms. The zinc trapping in turn was suspected to result in a cytosolic zinc deficiency, making zinc as an essential trace element unavailable for distinct organelles (e.g., ER, nucleus) or intracellular processes (secretory pathways, collagen modification, signaling pathways). Thus, in contrast to the original investigation by Giunta et al. [2008], the cytosolic zinc deficiency instead of the zinc overload in the ER was postulated as the main pathophysiological feature [Jeong et al. 2012]. The authors suggested the low cytosolic zinc concentrations to be involved in the alteration of TGF $\beta$ and bone morphogenetic protein signaling.

Hirose et al. [2015] further investigated SCD-EDS pathogenesis in the mouse model. They showed that a reduction of ZIP13 in fibroblasts of the knockout mice resulted in an alteration of ECM composition speculating that a decrease of decorin in the ECM may be responsible for the observed skin fragility and altered TGF $\beta$ levels. While the measured decorin values were insignificant, other ECM molecules with zinc-binding sites (nidogen, laminin, fibronectin, and c-krox), zinc-related interactions (e.g., decorin and fibrinogen), and their consequences on ECM-stability could be considered as potential candidates for future investigations [Reinhardt et

Mol Syndromol 2018;9:100-109 107 
al., 1993; Galéra et al., 1994; Ancsin and Kisilevsky, 1996; Dugan et al., 2003; Askari et al., 2007; Ragunathan et al., 2013].

Another study examined the functional role of the 2 known mutations in the SLC39A13 gene (9-bp deletion and G74D missense mutation) [Bin et al., 2014]. The authors demonstrated that both mutant proteins are degraded via the VCP-linked ubiquitin protease pathway and that inhibiting this degradation pathway restored protein expression and led to improved zinc homeostasis [Bin et al., 2014]. Therefore, inhibitors of proteasomedependent pathways were suggested as a potential therapeutic approach for SCD-EDS.

\section{Conclusion}

Given the high degree of overlap between myopathic and connective tissue disorders, our case presentation underlines the impact of NGS methods in clinically ambiguous cases. Here, we present for the first time concomitant myopathic findings in SCD-EDS, suggesting a broader clinical and pathophysiological presentation than originally suspected. However, the pathogenesis of SCD-EDS has not yet been fully understood, and addi- tional features may become apparent when more affected individuals have been discovered. Further analyses clarifying in which way mutations in the SLC39A13 gene affect skeletal muscle cells are warranted. Dissecting the genetic and molecular pathogenesis of rare diseases such as SCD-EDS may eventually pave the way for potential therapeutic approaches in the future [Bin et al., 2014].

\section{Acknowledgment}

We thank the family for taking part in the investigation as well as giving consent for publication of the data.

\section{Statement of Ethics}

The study was conducted in accordance with the Declaration of Helsinki. The affected boy and his parents gave their informed written consent and the study protocol was approved by the Ethics Committee of the Ruhr-University Bochum.

\section{Disclosure Statement}

The authors have no conflicts of interest to disclose.

\section{References}

Ancsin JB, Kisilevsky R: Laminin interactions important for basement membrane assembly are promoted by zinc and implicate laminin zinc finger-like sequences. J Biol Chem 271:68456851 (1996).

Askari J, Thornton DJ, Humphries J, Buckley PA, Humphries M: The alternatively spliced type III connecting segment of fibronectin is a zinc-binding module. Matrix Biol 26:485-493 (2007).

Beighton P, De Paepe A, Steinmann B, Tsipouras P, Wenstrup RJ: Ehlers-Danlos syndromes: revised nosology, Villefranche, 1997. EhlersDanlos National Foundation (USA) and Ehlers-Danlos Support Group (UK). Am J Med Genet 77:31-37 (1998).

Bin BH, Fukada T, Hosaka T, Yamasaki S, Ohashi $\mathrm{W}$, et al: Biochemical characterization of human ZIP13 protein: a homo-dimerized zinc transporter involved in the spondylocheiro dysplastic Ehlers-Danlos syndrome. J Biol Chem 286:40255-40265 (2011).

Bin BH, Hojyo S, Hosaka T, Bhin J, Kano H, et al: Molecular pathogenesis of spondylocheirodysplastic Ehlers-Danlos syndrome caused by mutant ZIP13 proteins. EMBO Mol Med 6: 1028-1042 (2014).
Brady AF, Demirdas S, Fournel-Gigleux S, Ghal N, Giunta C, et al: The Ehlers-Danlos syndromes, rare types. Am J Med Genet C Semin Med Genet 175:70-115 (2017).

Camacho Vanegas O, Bertini E, Zhang RZ, Petrini S, Minosse $\mathrm{C}$, et al: Ullrich scleroatonic muscular dystrophy is caused by recessive mutations in collagen type VI. Proc Natl Acad Sci USA 98:7516-7521 (2001).

Castori M, Voermans NC: Neurological manifestations of Ehlers-Danlos syndrome(s): a review. Iran J Neurol 13:190-208 (2014).

Chun S, Fay JC: Identification of deleterious mutations within three human genomes. Genome Res 19:1553-1561 (2009).

De Paepe A, Malfait F: The Ehlers-Danlos syndrome, a disorder with many faces. Clin $\mathrm{Ge}$ net 82:1-11 (2012).

Dugan TA, Yang VW, McQuillan DJ, Höök M: Decorin binds fibrinogen in a $\mathrm{Zn}^{2+}$-dependent interaction. J Biol Chem 278:1365513662 (2003)

Fukada T, Civic N, Furuichi T, Shimoda S, Mishima K, et al: The zinc transporter SLC39A13/ ZIP13 is required for connective tissue development; its involvement in BMP/TGF-beta signaling pathways. PLoS One 3:e3642 (2008).
Fukada T, Hojyo S, Furuichi T: Zinc signal: a new player in osteobiology. J Bone Miner Metab 31:129-135 (2013).

Galéra P, Musso M, Ducy P, Karsenty G: c-Krox, a transcriptional regulator of type I collagen gene expression, is preferentially expressed in skin. Proc Natl Acad Sci USA 91:9372-9376 (1994).

Giunta C, Elçioglu NH, Albrecht B, Eich G, Chambaz C, et al: Spondylocheiro dysplastic form of the Ehlers-Danlos syndrome - an autosomal-recessive entity caused by mutations in the zinc transporter gene SLC39A13. Am J Hum Genet 82:1290-1305 (2008).

Hara T, Takeda TA, Takagishi T, Fukue K, Kambe T, Fukada T: Physiological roles of zinc transporters: molecular and genetic importance in zinc homeostasis. J Physiol Sci 67: 283-301 (2017).

Hirano T, Murakami M, Fukada T, Nishida K, Yamasaki S, Suzuki T: Roles of zinc and zinc signaling in immunity: zinc as an intracellular signaling molecule. Adv Immunol 97:149176 (2008).
108

Mol Syndromol 2018;9:100-109

DOI: $10.1159 / 000485881$
Dusanic/Dekomien/Lücke/Vorgerd/Weis/ Epplen/Köhler/Hoffjan 
Hirose T, Ogura T, Tanaka K, Minaguchi J, Yamauchi T, et al: Comparative study of dermal components and plasma TGF- $\beta 1$ levels in Slc39a13/Zip13-KO mice. J Vet Med Sci 77: 1385-1389 (2015).

Jackson MJ: Physiology of zinc: general aspects, in Mills CF (ed): Zinc in Human Biology, pp 1-4 (Springer, London 1989).

Jeong J, Eide DJ: The SLC39 family of zinc transporters. Mol Aspects Med 34:612-619 (2013).

Jeong J, Walker JM, Wang F, Park JG, Palmer AE, et al: Promotion of vesicular zinc efflux by ZIP13 and its implications for spondylocheiro dysplastic Ehlers-Danlos syndrome. Proc Natl Acad Sci USA 109:E3530-3538 (2012).

Kambe T, Fukue K, Ishida R, Miyazaki S: Overview of inherited zinc deficiency in infants and children. J Nutr Sci Vitaminol (Tokyo) 61 Suppl:S44-46 (2015a).

Kambe T, Tsuji T, Hashimoto A, Itsumura N: The physiological, biochemical, and molecular roles of zinc transporters in zinc homeostasis and metabolism. Physiol Rev 95:749-784 (2015b).

Kimura T, Kambe T: The functions of metallothionein and ZIP and ZnT transporters: an overview and perspective. Int J Mol Sci 17:336 (2016).

Kirk JA, Ansell BM, Bywaters EG: The hypermobility syndrome. Musculoskeletal complaints associated with generalized joint hypermobility. Ann Rheum Dis 26:419-425 (1967).

Kirschner J, Hausser I, Zou Y, Schreiber G, Christen $\mathrm{HJ}$, et al: Ullrich congenital muscular dystrophy: connective tissue abnormalities in the skin support overlap with Ehlers-Danlos syndromes. Am J Med Genet A 132A:296-301 (2005).
Malfait F, Francomano C, Byers P, Belmont J, Berglund B, et al: The 2017 international classification of the Ehlers-Danlos syndromes. Am J Med Genet C Semin Med Genet 175: 8-26 (2017).

Mattle H, Mummenthaler M: Myopathien, in Mattle H, Mummentaler M (eds): Neurologie, ed 13, pp 632-678 (Georg Thieme Verlag KG, Stuttgart 2013).

Nakajima M, Mizumoto S, Miyake N, Kogawa R, Iida A, et al: Mutations in B3GALT6, which encodes a glycosaminoglycan linker region enzyme, cause a spectrum of skeletal and connective tissue disorders. Am J Hum Genet 92: 927-934 (2013).

Nigro V, Savarese M: Next-generation sequencing approaches for the diagnosis of skeletal muscle disorders. Curr Opin Neurol 29:621627 (2016).

Okajima T, Fukumoto S, Furukawa K, Urano T: Molecular basis for the progeroid variant of Ehlers-Danlos syndrome. Identification and characterization of two mutations in galactosyltransferase I gene. J Biol Chem 274:2884128844 (1999).

Ragunathan P, Sridaran D, Weigel A, Shabayek S, Spellerberg B, et al: Metal binding is critical for the folding and function of laminin binding protein, Lmb of Streptococcus agalactiae. PLoS One 8:e67517 (2013).

Ravenscroft G, Davis MR, Lamont P, Forrest A, Laing NG: New era in genetics of early-onset muscle disease: breakthroughs and challenges. Semin Cell Dev Biol 64:160-170 (2017).

Reinhardt D, Mann K, Nischt R, Fox JW, Chu ML, et al: Mapping of nidogen binding sites for collagen type IV, heparan sulfate proteoglycan, and zinc. J Biol Chem 268:10881-10887 (1993).
Royce PM, Steinmann B: Connective Tissue and Its Heritable Disorders: Molecular, Genetic, and Medical Aspects, ed 2, pp 431-523 (Wiley-Liss, New York 2002).

Savarese M, Di Fruscio G, Torella A, Fiorillo C, Magri F, et al: The genetic basis of undiagnosed muscular dystrophies and myopathies: results from 504 patients. Neurology 87:7176 (2016).

Savasta S, Merli P, Ruggieri M, Bianchi L, Spartà MV: Ehlers-Danlos syndrome and neurological features: a review. Childs Nerv Syst 27: 365-371 (2011).

Schwarz JM, Cooper DN, Schuelke M, Seelow D: MutationTaster2: mutation prediction for the deep-sequencing age. Nat Methods 11:361362 (2014).

Taylor KM, Nicholson R: The LZT proteins; the LIV-1 subfamily of zinc transporters. Biochim Biophys Acta 1611:16-30 (2003).

Voermans NC, Bönnemann CG, Huijing PA, Hamel BC, van Kuppevelt TH, et al: Clinical and molecular overlap between myopathies and inherited connective tissue diseases. Neuromuscul Disord 18:843-856 (2008).

Voermans NC, van Alfen N, Pillen S, Lammens $M$, Schalkwijk J, et al: Neuromuscular involvement in various types of Ehlers-Danlos syndrome. Ann Neurol 65:687-697 (2009).

Yonekawa T, Nishino I: Ullrich congenital muscular dystrophy: clinicopathological features, natural history and pathomechanism(s). J Neurol Neurosurg Psychiatry 86:280-287 (2015).
Nonsense Mutation in SLC39A13

Presenting as Myopathy
Mol Syndromol 2018;9:100-109

DOI: $10.1159 / 000485881$ 